\title{
Real Time Alert System: A Disease Management System Leveraging Health Information Exchange
}

\author{
Vibha Anand ${ }^{1,2}$, Meena E. Sheley ${ }^{1}$, Shawn $\mathrm{Xu}^{1}$,Stephen M. Downs ${ }^{1,2}$ \\ ${ }^{1}$ Children's Health Services Research, Indiana University School of Medicine, IN \\ ${ }^{2}$ Regenstrief Institute for Health Care, Indianapolis, IN
}

\begin{abstract}
Background: Rates of preventive and disease management services can be improved by providing automated alerts and reminders to primary care providers (PCPs) using of health information technology (HIT) tools.
\end{abstract}

Methods: Using Adaptive Turnaround Documents (ATAD), an existing Health Information Exchange (HIE) infrastructure and office fax machines, we developed a Real Time Alert (RTA) system. RTA is a computerized decision support system (CDSS) that is able to deliver alerts to PCPs statewide for recommended services around the time of the patient visit. RTA is also able to capture structured clinical data from providers using existing fax technology. In this study, we evaluate RTA's performance for alerting PCPs when their patients with asthma have an emergency room visit anywhere in the state.

Results: Our results show that RTA was successfully able to deliver "just in time" patientrelevant alerts to PCPs across the state. Furthermore, of those ATADs faxed back and automatically interpreted by the RTA system, 35\% reported finding the provided information helpful. The PCPs who reported finding information helpful also reported making a phone call, sending a letter or seeing the patient for follow up care.

Conclusions: We have successfully demonstrated the feasibility of electronically exchanging important patient related information with the PCPs statewide. This is despite a lack of a link with their electronic health records. We have shown that using our ATAD technology, a PCP can be notified quickly of an important event such as a patient's asthma related emergency room admission so further follow up can happen in near real time.

\section{Introduction}

With the passing of the Patient Protection and Affordable Care Act of 2010, there is a greater thrust towards prevention and management of chronic conditions in primary care settings. However, the rates of delivery of preventive care and disease management services are suboptimal and there is much room for improvement in primary care settings. [1-3] One possible improvement is implementation of evidence based care guidelines in routine practice. [4] However, assessment and implementation of evidence based care guidelines in primary care 
settings requires a significant level of knowledge and expertise not only among clinicians but also the clinical staff. Additionally, there are many formidable clinical workflow challenges to overcome for successful implementation. For example, significant amounts of time are needed to assess which guidelines and services may be applicable for a given patient at each visit. [5]

In the past, health information technology (HIT) tools such as computerized clinical decision support systems (CDSS) coupled with electronic health records (EHR) have been effective for care guidelines implementation. The CDSS work by applying electronically coded care guideline recommendations, such as checking for drug dosing or drug-drug interaction, against patients' electronic health records (EHR) to alert or remind the physician. However, most past successes with CDSS are attributed to direct interfaces such as those enabled through computerized physician order entry (CPOE) or note writing and mostly in inpatient settings. [6] A reminder to the provider at the time of note writing or order entry is often too late in primary care settings as events frequently take place after the primary care provider (PCP) has completed the visit. Therefore, what is needed are HIT tools which monitor real or near real time events and are able to couple with CDSS to deliver "just-in-time" (JIT) information, at or around the time of the patient's visit. However, such tools are feasible when an advanced infrastructure such as that for electronic delivery of information across a network of practices and providers exist, i.e. a Health Information Exchange (HIE) that is both functional and robust.

Our state has a robust and functionally advanced HIE, the Indiana Network for Patient Care (INPC) to provide the needed functionality. The INPC is a 16-year-old health information exchange operated by the Indiana Health Information Exchange (IHIE). Using standards, interoperability, and the interchange of clinical data for clinical, public health, and research purposes, investigators at the Regenstrief Institute created the INPC in 1995. The INPC includes clinical data from 45 hospitals, as well as from public health departments, laboratories, and imaging centers, and a few large-group practices closely tied to hospital systems. The INPC is continuing to expand. The HIE data repository carries over 4 billion pieces of clinical data, including over 79 million text reports. In addition to data from clinical institutions, the INPC also receives data from healthcare payers. The system is used for patient care, public health, and research.

In the INPC, events such as patient registration or discharge generate standard health level 7 (HL7) messages. The HL7 messages are delivered from participating practices and hospitals from across the state to the INPC. [7] The existing near real time delivery of standard HL7 messages to the INPC on events such as patient registration or discharge creates unique opportunity for improving care processes [8], for example, the registration messages from INPC may be used to trigger alerts and reminders to PCPs in order to provide evidence based preventive and disease management services that are due at a patient's visit. Additionally when coupled with a CDSS, the infrastructure can connect PCPs and practices from across the state for patients' follow up care [9, 10] perhaps using a JIT information approach, for example, the reminders and alerts to PCPs can be customized based on patient's previous labs and test results from their EHR.

However, even with an advanced infrastructure such as the INPC, there are practical limitations in implementing automated event monitoring and alerting systems in practice. One 
such crucial limitation is the delivery of information to the last mile i.e., to a practice in the field and back from it to the HIE. Much like the rest of the country [11], a majority of practices that participate in data sharing into INPC do not employ EHR in routine practice and therefore are not amenable for electronic delivery of information. Additionally, their current participation in the HIE consists of either receiving results through office fax machines or by logging on to HIE portal to download this information. Thus, despite our advanced infrastructure, a lack of a functional bi-directional interface makes it unfeasible to implement any automated event monitoring or alerting solution. Unless the last mile limitation is addressed in some way, our current infrastructure may be under-utilized to improve delivery rates of preventive care and disease management services across the state.

Therefore in the past two years, our research group has developed a mechanism for implementing a bi-directional interface between the HIE and practices in the field using existing fax technology. We chose to use fax as a medium for receiving data from practices in the field for two reasons. First and foremost fax machines are ubiquitous in physician offices and are already being used in patient care, making them easily accessible to securely exchange Protected Health Information (PHI). [12] Secondly any alternative electronic media use such as an email would not pass HIPAA legislation's [13] PHI security criteria, unless a institutional email system capable of exchanging secure messages electronically was established across all practices. [14] As most practices in our healthcare system reside outside our institutional email system, an email would not have been a secure way for receiving PHI and was not considered HIPAA safe for this study. Moreover, our fax interface which is described in detail elsewhere [15] has been previously used for linking clinicians in our pediatric practices to the state department of public health for test results of newborn screens. In this paper, we describe our use of this interface to develop and evaluate an event monitoring CDSS, the Real Time Alert (RTA) system to coordinate follow up care of an asthma related emergency room (ER) admission across practices throughout Indiana.

\section{Methods}

\section{The RTA System}

The RTA system is a CDSS that intercepts incoming patient discharge events from INPC participating practices to generate alerts and reminders based on patient's record in the EHR. RTA draws on the rich legacy of data already contained within INPC. [16] Using these data, RTA is able to generate reminder alerts that apply to a particular visit for a given patient and deliver those results to PCPs and practices across the state in real time or near real time. RTA is able to deliver these reminder alerts to the PCPs using INPC's existing document delivery service, DOCS4DOCS ${ }^{\circledR}$ or D4D. [17] D4D is a messaging service that securely and electronically delivers documents, such as laboratory results, in PDF format to providers across the state using their National Provider Index (NPI) number. Although in a few cases D4D delivers messages directly into an EHR, in most cases, the documents are sent to a D4D secure email box or fax machine.

The asthma module of the RTA system is designed to alert PCPs if their patients' have any asthma related ER admission. International Classification of Diseases-ninth revision (ICD9) is used for the purposes of detecting patient's ER visit as follows. The asthma module 
monitors HL7 messages from ERs into the INPC that include a discharge diagnosis of 493.* (asthma). When such a message is detected, RTA alerts the patient's PCP so that he or she can arrange a return visit, for example, to step up the asthma therapy in accordance with published asthma care guidelines. $[18,19]$ In addition, to reduce the burden of care coordination, the PCP alert is also accompanied by a pre-printed letter that the practice can send to the patient. Finally, RTA also provides a form on which PCPs and practices across the state can indicate receipt and use of the alert and update the INPC by checking boxes and faxing the form. The forms are then read by RTA, using optical character recognition and optical mark recognition (OCR/OMR) software.

The RTA system was initiated on January $9^{\text {th }} 2012$ and has been operational for asthma care follow up since then. The data we evaluate for this study are from June 2012 to November 2012. Below, we describe RTA's workflow, but first we briefly describe the Adaptive Turnaround Documents (ATAD) interface used for this study.

\section{Adaptive Turnaround Documents (ATAD)}

Adaptive Turnaround Documents (ATAD) are printable forms that are dynamically generated by the RTA system. [20] (Figure 1) The ATAD in Figure 1 is for notifying the PCP of their patient's previous ER admission. When the RTA system detects an HL7 message from an ER with an asthma discharge diagnosis, rules trigger the generation of the physician alert. The rules in the RTA system are encoded as Arden Syntax medical logic modules (MLM) [21-23] and the alerts and reminders are generated as ATAD.

ATADs contain patient specific information for the PCP. The ATADs are sent to D4D queue and delivered to PCPs either using faxes or electronically. Depending on practice preference, the personnel in PCP practices are able to view and print the ATADs or are able to view them by logging on to the secure HIE portal.

Specifically, the two ATADs that get generated by the RTA system for asthma follow up care are -1) ER admission notification ATAD and 2) a preprinted letter ATAD that is addressed to the patient as a convenience for the practice. They are described below.

\section{- ER Admission notification ATAD}

This ATAD contains information about patient's ER admission - date, time and location. (Figure 1) It also contains contact information about the patient and the provider (PCP). This ATAD notifies the PCP of the patient's ER visit so that the PCP may decide how best to respond to the information. The system does not generate specific recommendations but only alerts the PCP to follow up.

In response to this ATAD, the physician can respond to the RTA system by checking a box indicating any or all of the following: "This is not my patient", "We have sent this patient a letter", "We have seen this patient since the ER admission", or "We have called this patient". For example in Figure 1, the PCP responded that they have sent the letter and also called the patient regarding their ER admission. 
To receive this and similar ATADs securely, the RTA system is setup with a fax line. PCPs are requested to return the ER admission notification ATAD to the RTA system by fax. (Figure 1 shows a received ATAD by fax) The returned ATADs are optically read and interpreted by a third party OCR/OMR software (www.verity.com) suite. Structured data captured from the check boxes on the returned forms is stored electronically by the RTA system.

\section{- Letter for the patient ATAD}

Included with the ER admission notification ATAD is an addressed letter that the physician may send to the patient, inviting him or her to come to the clinic for a follow-up visit. (Figure 2) This letter is provided only as a convenience to the practice so that it can be sent to the patient's home address with the PCP's approval. It includes both the PCP's and patient's name as well as an invitation to the patient to schedule a clinic visit. 
Real Time Alert System: A Disease Management System Leveraging Health Information Exchange

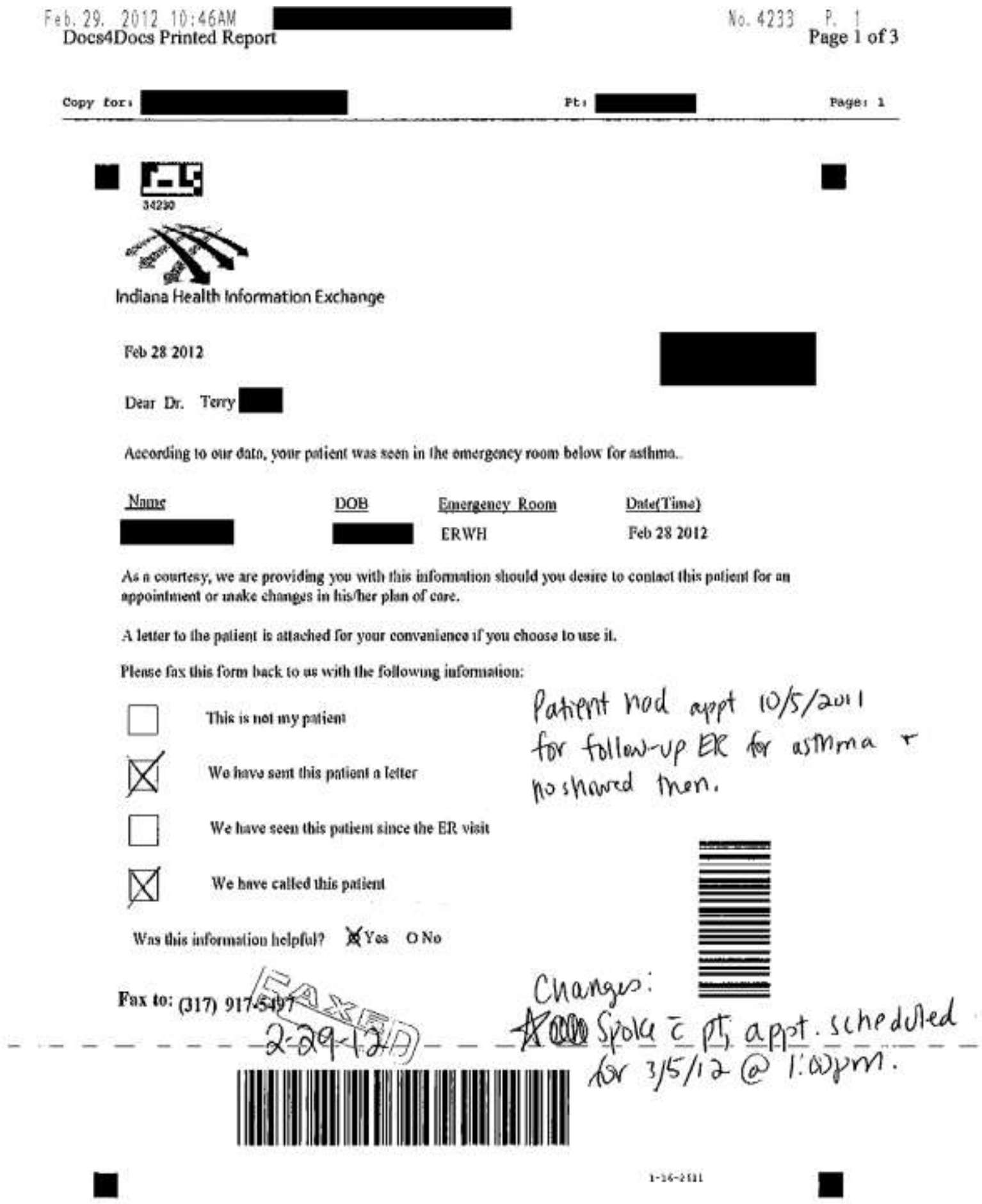

httos://docs4docs.ihie.oro/d4d orod/d4d?INBOXREVIEW SHOWDOCUMENT\&T D.. 02/29/2012

Figure 1: ER admission notification ATAD 
Real Time Alert System: A Disease Management System Leveraging Health Information Exchange

\section{-}

Mar 52012

From: Dr Martin

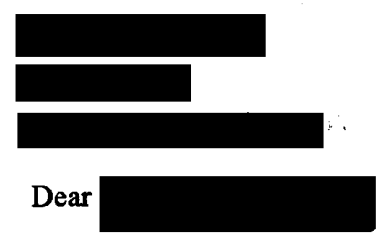

According to our records, you recently visited an emergency room for asthma. Changes in treatment plans are sometimes necessary for patients with asthma in order to prevent the need for emergency room visits.

.

Please call the office and schedule an appointment so we may discuss your current asthma treatment plan.

Sincerely,

Dr. Martin 


\section{The RTA Workflow}

The RTA workflow is described in Figure 3. When an HL7 message, containing a diagnosis of asthma (ICD-9 code - 493.*) in any valid segment of the HL7, for example DG1, FT1, or the OBX segment, arrives into the INPC from any ER, then our infrastructure is programmed to query an existing PCP attribution service to identify the PCP, using data within INPC. In brief, the PCP attribution service uses statistical methods to infer who the PCP is for a given patient and a description of those details are outside the scope of the current study.

If a PCP attribution is successful, an INPC process looks up the provider's NPI in a table that locates the PCP for a D4D delivery. The INPC process then constructs an outbound HL7 ADT message (Step 1 of Figure 3) to the RTA system to trigger the processes in the RTA system. The trigger message contains both the provider and the patient information - the NPI of PCP in the PV1 segment (as required by the D4D service for message delivery) and the patient's information in the patient identifier (PID) segment. On receiving the trigger message, RTA generates the two previously described ATADs (Figures 1 and 2).

The ATADs are generated as PDF documents and prepared for delivery to the inbound queue of the D4D service as follows. An outbound HL7 OBR message is constructed with the provider and the patient information transferred from the trigger message; the two ATADs are base-64 encoded (as required by D4D) and attached to the message (in steps 4 and 5 of Figure 3) and finally an exporter task in the RTA system sends the message to IHIE's D4D inbound queue.

The D4D service periodically polls its inbound queue and delivers the queued messages to the participating providers and practices across the state (as listed in the queued messages). The messages are delivered to the PCP's D4D inbox by default, though a PCP may also elect to receive the D4D messages by fax. Thus the message is delivered to the PCP's (that was indicated in the hl7 trigger message) D4D inbox informing them of their patient's recent ER admission for asthma care. All HL7 messages that are exchanged from RTA with INPC and D4D are protected behind institutional firewalls.

To indicate their follow up, PCPs are encouraged to complete and fax the ER admission notification form back to the fax line in the RTA system. The faxes are electronically read and data from them are extracted using OCR/OMR software. [20, 24] The structured data captured using OMR is then processed and deposited back to the INPC repository. These steps are detailed in steps $6-10$ of Figure 3. 


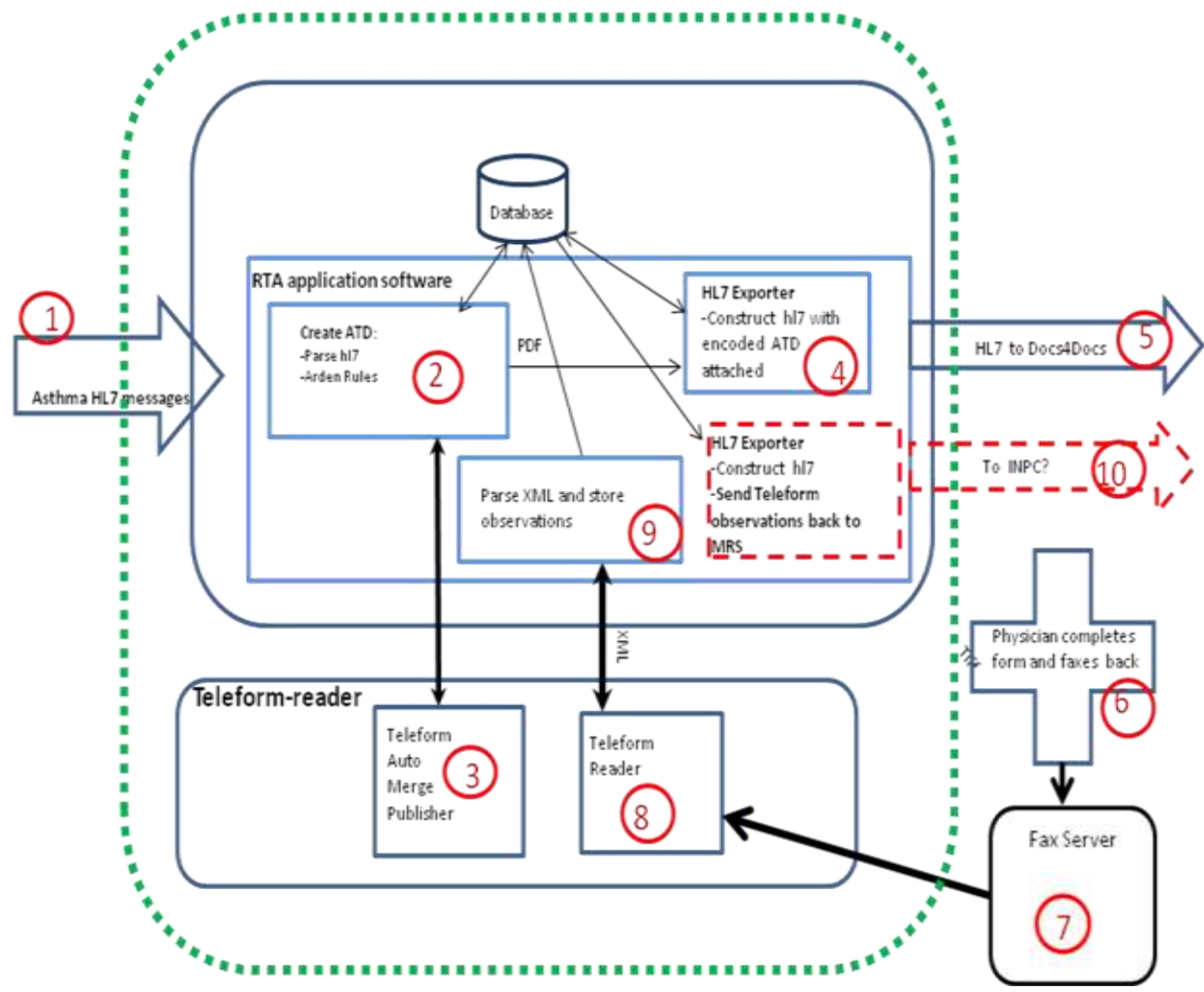

Figure 3: RTA Workflow

\section{Results}

During the five month study period (June 2012 to November 2012), 2120 trigger messages pertaining to an asthma related ER admission were received by the RTA system from INPC. Of these trigger messages, 1958 (92\%) messages were processed. One hundred and sixty two $(7 \%)$ trigger messages were not processed because they were either duplicates or were received in an invalid format. Of those processed, $676(35 \%)$ were missing a NPI needed to identify the PCP and, therefore, were not acted upon by the RTA system, and $7(<1 \%)$ were undeliverable. Therefore, of the processed trigger messages, $1275(65 \%)$ resulted in an ER admission notification to 538 unique PCPs across the state. (Figure 4) Of those PCPs who received an ER admission notification ATAD (Figure 1), 219 (41\%) received more than one, and $42(8 \%)$ received more than five notifications during the study. These notifications were either for the same or different patients suggesting that these practices see a high number of asthma patients who had asthma exacerbations. 
Collectively PCPs faxed $126(10 \%)$ ER admission notifications back to the RTA system. Of those, $31(25 \%)$ ATADs were unreadable by the computer using automated OMR methods due to misaligned or poor quality faxes. Of those PCPs who returned an ER admission notification, 79 (15\%) indicated they had taken action in response to the alerts.

Furthermore, the forms returned by the PCPs were analyzed by extracting data from RTA's database. The PCPs responses on these forms are not considered mutually exclusive for our analyses and the details of this analysis are in Figure 4. There were 95 (75\%) forms that were automatically interpretable using OMR methods by the computer. Of those $33(35 \%)$ indicated that the PCP found the provided information helpful and 19 (20\%) indicated that the PCP did not find the information helpful. However, $23(24 \%)$ returned forms indicated that the PCP made a phone call to follow up with their patient and 4 (4\%) forms also indicated that the PCP sent the attached letter to the patient in response to this notification. Ten $(11 \%)$ returned forms indicated that the PCP had seen the patient post their ER admission and $32(34 \%)$ forms indicated that the patient they were contacted for was not their patient.

The average time between when the RTA received a trigger message and when the PCP was notified with a message was $2.6 \mathrm{hrs}$. The average time from when the ER admission notifications were sent to the PCP to when they were returned to RTA was 4.6 days in this study.

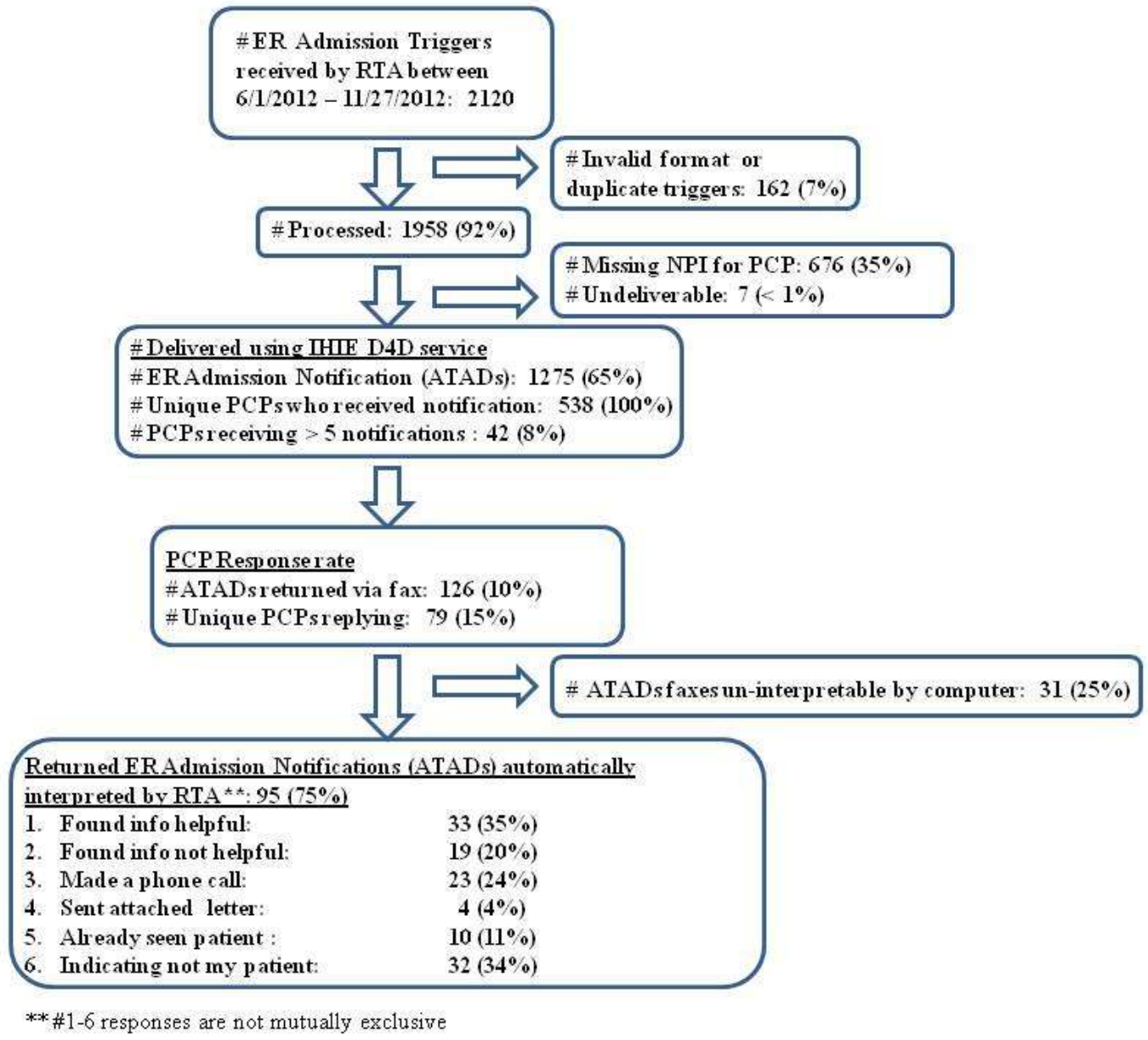

Figure 4: RTA Evaluation Results 


\section{Discussion}

In this study, we have demonstrated the feasibility of electronically exchanging important patient related information with primary care providers despite the lack of a link with their EHR. We have shown that using our ATAD technology, a PCP can be notified quickly of an important event such as a patient's asthma related ER admission so further action can be taken in near real time. We have also shown that, using an existing health information exchange and everyday office equipment like fax machines, alerts can be delivered to any PCP across the state with simple technology.

Our ability to interpret the value of these notifications to physicians is limited by both the poor response rate from the clinics we asked to fax us the forms and by limitations of our PCP attribution service. Experience in our HIE has shown that PCP attribution requires ongoing manual correction, usually by physicians indicating that a patient is not theirs. For example, our rates of "This is not my patient" response from PCPs dropped considerably (63\% to 34\%) when information was corrected. On visually examining the provider returned forms, we found that many indicated that they were the patient's specialist, for example an eye doctor or their gynecologist but not their PCP.

Nonetheless, based on the forms we did receive, we believe that when notified, PCPs find such information helpful more than a third of the time. Furthermore, the majority of PCPs receiving the information and responding actually reached out to their patients by making a phone call, emphasizing the nature of the alert and immediacy of follow up.

As with all such research, our methods have both advantages and limitations. The advantage of our approach is that primary care practices with only a fax machine are able to share data with the HIE. However, this approach does push the burden and complexities of sharing information to entities running HIE and systems like RTA. For example, though most faxes are computer interpretable without human intervention, still personnel are needed to verify and review faxes manually if a greater accuracy is desired and if all data that is exchanged needs to be captured.

\section{Conclusion}

Despite its drawbacks, we believe that the RTA bi-directional information exchange method using existing fax technology is both feasible and innovative for follow up care of chronic illness. It can also be used for management of many other chronic diseases such as diabetes or hypertension. We also believe it can bridge the last mile barrier for adoption and tight integration of EHRs across PCPs and practices. [15] Furthermore, our method provides a pragmatic mechanism for exchange of information until universal identifiers for linking patients in different EHR systems can be determined. [15, 25] 


\section{Corresponding Author}

Vibha Anand, PhD

Indiana University

Email: vanand@iupui.edu

\section{References}

1. Fani Marvasti F, Stafford RS. 2012. From sick care to health care - reengineering prevention into the u.s. System. N Engl J Med. 367(10), 889-91. http://dx.doi.org/10.1056/NEJMp1206230

2. Provost S, et al. 2010. Does Receiving Clinical Preventive Services Vary across Different Types of Primary Healthcare Organizations? Evidence from a Population-Based Survey. Health Policy. 6(2), 67-84.

3. Lalonde L, et al. 2012. Priorities for action to improve cardiovascular preventive care of patients with multimorbid conditions in primary care--a participatory action research project. Fam Pract. http://dx.doi.org/10.1093/fampra/cms021

4. Elson RB, Connelly DP. 1995. Computerized patient records in primary care. Their role in mediating guideline-driven physician behavior change. Arch Fam Med. 4(8), 698-705. http:// dx.doi.org/10.1001/archfami.4.8.698

5. Yarnall KS, et al. 2003. Primary care: is there enough time for prevention? Am J Public Health. 93(4), 635-41. http://dx.doi.org/10.2105/AJPH.93.4.635

6. Dexter PR, et al. 2001. A computerized reminder system to increase the use of preventive care for hospitalized patients. N Engl J Med. 345(13), 965-70. http://dx.doi.org/10.1056/NEJMsa010181

7. Overhage J, McDonald CJ, Suico JG. 2000. The regenstrief medical record system 2000:Expanding the breadth and depth of a community wide EMR. Proc AMIA Symp. $\bullet \bullet, 1173$. 8. Grannis SJ, et al. 2005. How disease surveillance systems can serve as practical building blocks for a health information infrastructure: the Indiana experience. AMIA Annu Symp Proc. •••, 286-90. 9. Grossman, J.M., K.L. Kushner, and E.A. November, Creating sustainable local health information exchanges: can barriers to stakeholder participation be overcome? Res Briefs, 2008(2): p. 1-12. 10. Chang KC, et al. 2010. Enhancing laboratory report contents to improve outpatient management of test results. J Am Med Inform Assoc. 17(1), 99-103. http://dx.doi.org/10.1197/jamia.M3391 11. Kemper AR, Uren RL, Clark SJ. 2006. Adoption of electronic health records in primary care pediatric practices. Pediatrics. 118(1), e20-24. http://dx.doi.org/10.1542/peds.2005-3000 12. American Health Information Management Association. Facsimile Transmission of Health Information (Updated). 2001; Available from: http://library.ahima.org/xpedio/groups/public/ documents/ahima/bok1 031811.hcsp?dDocNa me=bok1 031811.

13. US Department of Health and Human Services. H. The Health Insurance Portability and Accountability Act of 1996 (HIPAA) Privacy and Security Rules. 1996; Available from: http:// www.hhs.gov/ocr/privacy/index.html.

14. Gerstle RS. 2004. E-mail communication between pediatricians and their patients. Pediatrics. 114(1), 317-21. http://dx.doi.org/10.1542/peds.114.1.317 
15. Downs SM. 2011. A.V., Sheley ME, Grannis SJ, The Last Mile: Using Fax Machines to Exchange Data between clinicians and Public Health. Online J Public Health Inform. 3(3). http://dx.doi.org/10.5210/ojphi.v3i3.3892

16. Biondich PG, Grannis SJ. 2004. The Indiana network for patient care: an integrated clinical information system informed by over thirty years of experience. J Public Health Manag Pract. Suppl, S81-86. http://dx.doi.org/10.1097/00124784-200411001-00013

17. Barnes M. 2007. Lessons learned from the implementation of clinical messaging systems. AMIA Annu Symp Proc. $\cdots, 36-40$.

18. National Asthma Education and Prevention Program. Expert Panel Report 3: Guidelines for the Diagnosis and Management of Asthma. National Heart, Lung, and Blood Institute, National Institutes of Health, 2007.

19. National Heart. L., and Blood Institute, National Asthma Education and Prevention Program, Expert Panel Report 2: Guidelines for the Diagnosis and Management of Asthma. National Institute of Health, 1997.

20. Biondich PG, et al. 2003. Using adaptive turnaround documents to electronically acquire structured data in clinical settings. AMIA Annu Symp Proc. $\cdots$, , 86-90.

21. Downs S, et al. 2006. Using Arden Syntax and adaptive turnaround documents to evaluate clinical guidelines. AMIA Annu Symp Proc. $\cdots, 2$, 214-18.

22. Jenders RA, Corman R, Dasgupta B. 2003. Making the standard more standard: a data and query model for knowledge representation in the Arden syntax. AMIA Annu Symp Proc. .••, 323-30.

23. Jenders RA, et al. 1995. Medical decision support: experience with implementing the Arden Syntax at the Columbia-Presbyterian Medical Center. Proc Annu Symp Comput Appl Med Care. $\cdots, 169-73$.

24. Biondich PG, et al. 2002. A modern optical character recognition system in a real world clinical setting: some accuracy and feasibility observations. Proc AMIA Symp. $\cdots$, , 56-60. 25. Zhu VJ, et al. 2009. An empiric modification to the probabilistic record linkage algorithm using frequency-based weight scaling. J Am Med Inform Assoc. 16(5), 738-45. http:// dx.doi.org/10.1197/jamia.M3186 